\title{
Snapshots of a genetic cut-and-paste
}

transposition now reveal the remarkable conformational changes in the protein during this process.

Perhaps most notably, the authors find that the $\mathrm{ZnB}$ wings move constantly: they unfurl when transposon DNA first arrives, and then close and open again during the rest of the process. This 'flapping' accompanies some impressive DNA acrobatics, which brings different DNA parts into the protein's core for ordered cleavage and joining. Remarkably, the $\mathrm{ZnB}$ domains help to capture not only the transposon, but also the target molecules into which Transib will be inserted - first opening to make space for the molecules, and then closing to plug the region of the target DNA into which Transib will be integrated into the core. The structures also show that the end section of the protein (the carboxy-terminal tail; CTT) contains three short $\alpha$-helices that form an accordion-like structure, which connects the moving wings to the complex's 'body'. Previously reported transposase structures $^{6-11}$ have revealed similar overall features, but the movements in the Transib transposase are much more extensive.

RAG protein complexes consist of two transposase-like RAG1 proteins and two RAG2 proteins. In these complexes, $\mathrm{ZnB}$ is present in RAG1, but is more fixed than in the Transib transposase; and no part is present ${ }^{13}$ that is analogous to CTT. RAG2, which is essential in $\mathrm{V}(\mathrm{D}) \mathrm{J}$ recombination but absent in most transposases, sits above the wings of RAG1 and holds a large part of the DNA ${ }^{12}-$ much as $\mathrm{ZnB}$ and CTT do in the Transib transposase.

Unlike transposases, the RAG complex cuts This process is called $\mathrm{V}(\mathrm{D}) \mathrm{J}$ recombination, and equips immune cells with a diverse set of sensors that can recognize many threats ${ }^{3}$. On page 540, Liu et al. ${ }^{4}$ report a series of structures of a transposase that is an ancestor ${ }^{5}$ of RAG, casting light on the evolutionary history of these proteins.

Transposases must recognize several DNA sites, and then cut and join them in the proper order $^{6}$. To understand this multi-step process, we need to visualize the structures of the molecular machinery involved at all stages, which is a major technical challenge. Liu et al. have now drawn on a powerful combination of two techniques - X-ray crystallography and single-particle cryo-electron microscopy - to picture several steps of transposition in remarkable detail, thereby providing a molecular 'movie' of the process.

The authors' achievements build on many years of structural studies of transposases ${ }^{7-11}$ and $\mathrm{RAG}^{12-15}$, providing an increasingly complete view of their functions and helping to connect the dots between the 'selfish' DNA rearrangements of transposases and the essential functions that evolved from them. Transposases are now known to have a catalytic core unit and diverse extra parts that bind formed mainly from a protein region called the zinc-binding $(\mathrm{ZnB})$ domain. The wings provide many of the interactions with the DNA, which forms the 'antennae' (Fig. 1).

As with many transposons, Transib moves by a cut-and-paste process: its transposase cleaves it out of the genome, using a catalytic core present in many transposases, and inserts it elsewhere in the genome ${ }^{16}$. Liu and co-workers' structures of five steps in Transib

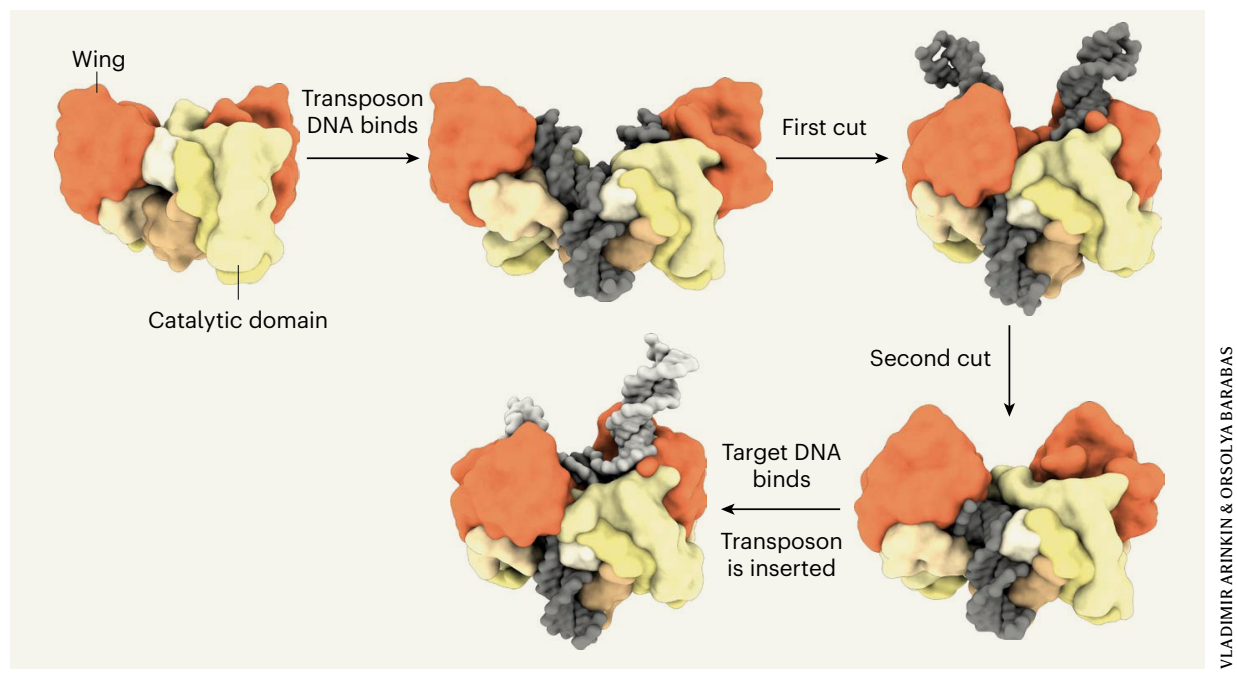

Figure 1 | Flapping of a transposase complex. Liu et al. ${ }^{4}$ report a series of structures of the transposase enzyme that mediates the movement of the Transib transposon (a parasitic genetic element) within genomes; the first structure shown was obtained using X-ray crystallography, and the others were obtained using single-particle cryo-electron microscopy. The transposase forms a dimeric complex that is roughly moth-shaped. The 'wings' unfurl to capture transposon-containing DNA, and then close again as the catalytic domain makes the first cut to cleave the transposase out of the DNA. The wings open again after the second cut, allowing target DNA (the DNA into which the transposon will be inserted) to be captured and the transposon to be inserted. The DNA sequences differ in length in some of the panels. 
and removes the distinct DNA sequences found between the $\mathrm{V}, \mathrm{D}$ and J gene segments, tightly coordinating the process to ensure that different types of segment are subsequently connected. In cells, RAG also largely stops the removed DNA from being reinserted elsewhere in the genome, to prevent potentially harmful changes to the genetic code. But how did these functions evolve? A logical proposal implicates RAG2, but a recent study ${ }^{11}$ of ProtoRAG - a relative of RAG found in invertebrates that contains RAG 2 but still acts as a transposase - shows that things are more complicated. Elements in both RAG1 and RAG2 help to coordinate DNA cleavage and prevent insertion.

Liu and colleagues' findings cast fresh light on the role of RAG2, showing that it carries out many of the functions of $\mathrm{ZnB}$, but increases the rigidity of the whole RAG complex, compared with that of the transposase complex. It binds the DNA at the $V$, D and J segments more tightly than $\mathrm{ZnB}$ binds at the transposon, and does not undergo such large conformational changes (which can require a lot of energy, and thus reduce efficiency). This increased rigidity and tight binding might help to ensure the strict molecular coordination required for $\mathrm{V}(\mathrm{D}) \mathrm{J}$ recombination. It might also prevent release of cleaved DNA segments and/or stop the wings from reopening to accept any other DNA molecules - thereby preventing removed DNA from being reinserted elsewhere in the genome. If the wings do not open, then any incoming DNA would have to bend itself to an angle of about $150^{\circ}$ before entering the protein, which is not easily done.

Note that Liu et al. were not able to directly observe the structure of the transposase in complex with intact target DNA. It therefore remains to be seen whether target DNA first binds to the transposase in a relaxed form and is then forced into a severe $150^{\circ}$ bend. The authors also did not observe a complex in which the transposase binds intact transposon DNA such that the catalytic core is close enough to the ends to cleave them; instead, the authors observed intact transposon DNA bound with its ends away from the catalytic centre. In RAG, a large twist in the DNA is needed to position its breakpoints accurately for the cuts ${ }^{14}$. A similar twist might occur in Transib, but other explanations are also possible.

Efforts are now needed to define the exact functions of RAG2. Curiously, the cell-free RAG complex can readily insert excised DNA into another DNA molecule ${ }^{17,18}$ (a target DNA). Structures of RAG with a bound target DNA must therefore be obtained - ideally, both with the intact target and after insertion. These structures will show whether the target DNA becomes as sharply bent as it does in the Transib transposase, and reveal how RAG 2 affects the binding of target
DNA and its insertion of excised DNA.

Other proteins might be needed to promote the function of RAG. This possibility has previously been investigated, but the availability of new structures and methods provides further opportunities for research. For example, large molecular assemblies can now be studied inside cells using a technique called electron tomography ${ }^{19}$, and molecular interactions can be probed with advanced massspectrometry methods ${ }^{20}$. Analysis of genomic data from different species will also be helpful in identifying ancestors of RAG proteins other than ProtoRAG and the Transib transposase, and thereby exploring their evolutionary history. Such research will help to explain how parasitic genetic elements can be repurposed for crucial biological functions.
Orsolya Barabas is in the Structural
and Computational Biology Unit,
European Molecular Biology Laboratory, 69117 Heidelberg, Germany.
e-mail: barabas@embl.de

1. Lander, E. S. et al. Nature 409, 860-921 (2001).

2. Bourque, G. et al. Genome Biol. 19, 199 (2018)

3. Jones, J. M. \& Gellert, M. Immunol Rev. 200, 233-248 (2004).

4. Liu, C., Yang, Y. \& Schatz, D. G. Nature 575, 540-544 (2019)

5. Kapitonov, V. V. \& Jurka, J. PLoS Biol. 3, e181 (2005).

6. Hickman, A. B. \& Dyda, F. Microbiol. Spectr. 3 MDNA3-0034-2014 (2015).

7. Davies, D. R., Goryshin, I. Y., Reznikoff, W. S. \& Rayment, I. Science 289, 77-85 (2000)

8. Richardson, J. M., Colloms, S. D., Finnegan, D. J. \& Walkinshaw, M. D. Cell 138, 1096-1108 (2009).

9. Hickman, A. B. et al. Nucleic Acids Res. 46, 10286-10301 (2018).

10. Montaño, S. P., Pigli, Y. Z. \& Rice, P. A. Nature 491, 413-417 (2012).

11. Zhang, Y. et al. Nature 569, 79-84 (2019).

12. Ru, H. et al. Cell 163, 1138-1152 (2015).

13. Kim, M.-S. et al. Mol. Cell 70, 358-370 (2018).

14. Ru, H. et al. Nature Struct. Mol. Biol. 25, 732-742 (2018).

15. Kim, M.-S., Lapkouski, M., Yang, W. \& Gellert, M. Nature 518, 507-511 (2015).

16. Chen, S. \& Li, X. Gene 408, 51-63 (2008)

17. Hiom, K., Melek, M. \& Gellert, M. Cell 94, 463-470 (1998).

18. Agrawal, A., Eastman, Q. M. \& Schatz, D. G. Nature 394, 744-751 (1998).

19. Mahamid, J. et al. Science 351, 969-972 (2016).

20. Smits, A. H. \& Vermeulen, M. Trends Biotechnol. 34 825-834 (2016).

\section{Astrophysics}

\section{Extreme emission seen from $\gamma$-ray bursts}

Bing Zhang

Cosmic explosions called $\gamma$-ray bursts are the most energetic bursting events in the Universe. Observations of extremely high-energy emission from two $\gamma$-ray bursts provide a new way to study these gigantic explosions. See p.455, p.459 \& p.464

Astrophysical explosions known as $\gamma$-ray bursts (GRBs) can release in one second the amount of energy that the Sun will produce in its entire lifetime ${ }^{1}$. The emission from GRBs covers a broad stretch of the electromagnetic spectrum and occurs in two stages: the prompt-emission phase and the afterglow phase. The main emission mechanism is thought to be synchrotron radiation, whereby the gyration of energetic electrons around magnetic-field lines releases photons. Until now, emission from GRBs has been observed only at energies below 100 gigaelectronvolts $(\mathrm{GeV})$. Three papers in this issue $\mathrm{e}^{2-4}$ report observations of $\gamma$-rays that have energies above $100 \mathrm{GeV}$ from two bright GRBs, dubbed GRB 190114C and GRB 180720B.

The Major Atmospheric Gamma Imaging Cherenkov (MAGIC) Collaboration ${ }^{2}$ (page 455) detected photons in the teraelectronvolt range $\left(1 \mathrm{TeV}\right.$ is $10^{3} \mathrm{GeV}$ ) from GRB $190114 \mathrm{C}$, using the MAGIC telescopes at La Palma, Spain. The first detections started about one minute after the burst triggered NASA's two spaceborne GRB detectors: the Burst Alert Telescope on board the Swift satellite and the Gamma-ray Burst Monitor on board the Fermi satellite. The high-energy photons continued to rain down on the MAGIC telescopes for about 20 minutes, with the flux decreasing rapidly over this time. The MAGIC Collaboration and colleagues $^{3}$ (page 459) detected this GRB using several other ground-based and space-borne telescopes. When combined with the MAGIC data, this rich data set allowed the authors to model the event comprehensively and study how the TeV emission was produced.

Abdalla et al. ${ }^{4}$ (page 464) detected photons of energies above $100 \mathrm{GeV}$ (but below $1 \mathrm{TeV}$ ) from GRB 180720B, using the High Energy Stereoscopic System (HESS) array of telescopes in Namibia. Although these photons were lower in energy and fewer in number than those observed from GRB 190114C, they were detected from deep in the afterglow phase (10 hours after the GRB was triggered and 